\title{
Extrapulmonary Tuberculosis masquerading as chest wall malignancy: It just never ceases to surprise us!
}

\author{
Mousa Hussein ${ }^{1}$, Abdalhadi Ahmed ${ }^{1}$, Anam Elarabi ${ }^{1}$, Ibrahim Rasheed ${ }^{1}$, Abbas Alabbas ${ }^{1}$, \\ and Aisha Aladab ${ }^{1}$ \\ ${ }^{1}$ Hamad Medical Corporation
}

January 30, 2021

\begin{abstract}
With the emergence of the acquired immunodeficiency syndrome, we witnessed a higher incidence of disseminated and extrapulmonary tuberculosis. It poses a significant diagnostic challenge for the physicians; therefore, a high index of suspicion should be maintained. Here we present a case of isolated chest wall tuberculosis in an immunocompetent patient.

Extrapulmonary Tuberculosis masquerading as chest wall malignancy: It just never ceases to surprise us! Mousa Hussein* ${ }^{1}$, Ahmad Abdelhadi ${ }^{2}$, Anam Elarabi ${ }^{1}$, Ibrahim Rashid ${ }^{1}$, Abbas Alabbas ${ }^{1}$, Aisha Aladab ${ }^{1}$

${ }^{1}$ Pulmonary department, Hamad General hospital

${ }^{2}$ Internal medicine department, Hamad General Hospital

Corresponding Author: Mousa Hussein, MD, CABMS, MRCP Pulmonology Department, Hamad General Hospital, Hamad Medical Corporation, Box: 3050 Doha, Qatar. Email: mhussein11@hamad.qa

Conflict of Interest: None

Acknowledgment: Open access publication funded by the Qatar National Library.

Abstract:

With the emergence of the acquired immunodeficiency syndrome, we witnessed a higher incidence of disseminated and extrapulmonary tuberculosis. The infection sites commonly include lymph nodes, pleura, and osteoarticular areas, although any organ can be involved. Given the atypical presentation of the extrapulmonary disease, it poses a significant diagnostic challenge for the physicians; therefore, a high index of suspicion should be maintained, particularly where tuberculosis is endemic. Here we present a case of isolated chest wall tuberculosis in an immunocompetent patient.
\end{abstract}

\section{Keywords:}

Extrapulmonary Tuberculosis, Musculoskeletal Tuberculosis, Chest wall mass, Cold abscess, Rib fracture

\section{Introduction:}

Tuberculosis (TB) is an infectious disease caused by mycobacterium tuberculosis (MTB), which spread through the air and commonly affects the lungs. It has an increased incidence during the last few decades despite the effective management because of the emergence of the human immunodeficiency virus (HIV) and the wide use of immunosuppressive medications. (1) Tuberculosis's most common presentation is pulmonary infection; however, it can affect any organ in the body such as the pleura, central nervous system, lymphatic 
system, genitourinary system, and musculoskeletal system. (2) One of the rare presentations of the musculoskeletal system infection is chest wall involvement, representing around 2 percent of tuberculosis cases overall. (3) Herewith we present an interesting case of chest wall tuberculosis in a young immunocompetent patient.

\section{Case presentation:}

A 24-year-old male patient, who was previously healthy, presented to the Hamad general hospital complaining of progressive left-sided pleuritic chest pain lasting for a few weeks. He noticed two large lumps in front of his chest, precisely at the same site of the pain slowly increasing in size over the last four months. He experienced a loss of appetite and weight of eight kilograms over the same duration. The patient denied any history of cough, shortness of breath, fever, or night sweats. He was a lifelong nonsmoker, with no contact with sick people and no recent travel, with unremarkable family history. On examination, he was afebrile, maintaining normal oxygen saturation on ambient air. There were two anterior chest wall masses: the first above the left second rib, measuring $7 \times 7 \mathrm{~cm}$, and the second over the left costal margin measuring 10x10 $\mathrm{cm}$. The covering skin of both masses appeared normal with no draining sinus or tract. The lumps were mobile, cold, non-pulsatile, and not tender. Chest auscultation and examination of other relevant systems were unremarkable. His laboratory investigations showed hemoglobin of $11.4 \mathrm{~g} / \mathrm{dl}$, C-reactive protein of 65 mg/L, positive Quantiferon TB gold plus, and normal kidney and liver function tests (Table 1).

A chest x-ray showed airspace opacity noted in the left perihilar region (Figure 1). Chest computed tomography (CT) showed two lenticular-shaped collections noted in the left anterior chest wall. One is noted along the internal surface of the left third rib measures $19 \times 53 \mathrm{~mm}$ causing bone destruction. The other one is in the subcutaneous tissue measures $60 \times 33 \mathrm{~mm}$, with an enlarged left hilar lymph node showing peripheral enhancement and central hypodense necrotic area (Figure $2 \mathrm{~A}, \mathrm{~B}$ ). With associated underlying third rib destruction (Figure 3). Ultrasound examination of the lesion in the lower chest wall showed well defined hypoechoic area, with varying degrees of internal heterogeneity (Figure 4).

The patient failed to produce any sputum even after induction. The acid-fast bacilli smear (AFB) of the aspirated fluid from the chest wall swelling came negative, but the Polymerase Chain Reaction (PCR) came positive. The aspirated fluid culture grew a pan-sensitive Mycobacterium tuberculosis complex (MTB). We diagnosed him with primary chest wall tuberculosis and therefore started on first-line anti-tuberculosis medications. The patient traveled back to his home country and lost follow up with us.

\section{Discussion:}

Tuberculosis is a chronic infectious disease caused by M. tuberculosis. Every year, around ten million people are diagnosed with tuberculosis. Despite being a preventable and curable disease, it the world's top infectious killer. (2) The main risk factors for developing tuberculosis infection are poverty, undernutrition, diabetes, smoking, and immunocompromised conditions, namely, human immunodeficiency virus infection (HIV). (4)

The lungs are the primary site for tuberculosis infection, with various presentations including primary TB, reactivation TB, endobronchial TB, and tuberculoma. (4) The main pulmonary tuberculosis symptoms are fever, cough, difficulty breathing, anorexia, weight loss, and night sweats. However, in the advanced stage and the absence of proper treatment, complications may occur in the form of hemoptysis, pneumothorax, bronchiectasis, and extensive pulmonary destruction. (5)

Extrapulmonary tuberculosis refers to TB involving organs other than the lungs (e.g., pleura, lymph nodes, abdomen, genitourinary tract, skin, joints, bones, or meninges). It represented $15 \%$ of the 7.0 million incident cases notified in 2018, and the most typically reported sites were lymph node, pleura, and urogenital tuberculosis. (6)

Skeletal tuberculosis accounts for 2-6\% of all tuberculous infections, with the spine being the most commonly affected site. The chest wall involvement and ribs in skeletal tuberculosis are exceptionally uncommon, accounting for $<5 \%$ of bone and joint TB. It is twice more common in males than females, with typical age between 15-30 years, as in our patient. (7) 
TB abscesses of the chest wall are usually seen at the sternum margins and along the rib shafts. Three mechanisms have been described as responsible for the chest wall involvement, including a direct extension from the pleural or parenchymal disease, hematogenous dissemination, or lymphatic extension. (8) In our case, although the patient does not have any respiratory symptoms with no parenchymal involvement, the hilar and mediastinal lymph nodes enlargement with peripheral enhancement and central necrosis as seen on chest CT scan represents the possible focus for the chest wall involvement.

Chest wall tuberculosis usually presents insidiously as swelling and pain over the bone, with few constitutional symptoms, making the diagnosis difficult and delayed in most cases, averaging 4-28 months. Some patients may present with secondary infection complications, spontaneous fractures of the ribs or sternum, and compression or erosion of the large blood vessels. (9) Differential diagnosis of the chest wall swellings includes granulomatous diseases (sarcoidosis, Non-Tuberculosis mycobacterium), chronic infections (fungal or parasitic), and benign or malignant growth (fibrous dysplasia, osteoblastoma, chondral tumors, malignant bony or cartilaginous tumors, and metastasis). (10) Our patient presented with fourth months history of slowgrowing and painful anterior chest wall masses, weight loss, and anorexia with no evidence of complications.

The laboratory investigations for diagnosing chest wall TB are non-specific, including slightly raised inflammatory markers (C-reactive protein, wight blood cells, and serum ferritin), anemia of chronic disease, which were the same as found in our case. (11) The imaging modalities useful in chest wall TB are radiography, Ultrasound, and CT scan. The findings from these modalities help determine the degree of thoracic cage involvement and give a hint for the possible focus of involvement in the form of pleural, parenchymal, and mediastinal lymph nodes abnormalities. (12) Although the Magnetic Resonance Imaging (MRI) of the chest provides a lot of information about the soft tissues and degree of extension of the abscess; CT chest is considered the investigation of choice by many authors; as it is easily accessible, affordable, and provides detailed information almost as MRI chest. Typical CT findings in the chest wall TB include bone destruction, soft tissue masses crossing the fascial planes, with abscess and calcification, and underlying pleuro-parenchymal tubercular involvement. $(12,13)$ There is an increasing role for the Ultrasound to help localize the affected areas and the size of the collections. The abscesses usually appeared as hypoechoic areas, with varying degrees of internal heterogeneity. Bone fragments appeared as echogenic foci within these hypoechoic collections. (14)

Fine-needle aspiration (FNA) of the chest wall collection represents a simple, non-invasive way to get the diagnosis of tuberculosis. The aspirated material is usually sent for acid-fast bacilli (AFB) smear, PCR, and culture. The yield of AFB smear is low as it needs many bacteria; on the other hand, PCR requires a small number of bacteria and can detect drug resistance. In addition to identifying the sensitivity to the specific treatment, AFB cultures provide the advantage of knowing the organism species to exclude the Mycobacteria other than tuberculosis as a well-known cause of chest wall infections $(11,15)$. In our patient, PCR and culture confirmed the diagnosis of Mycobacterium Tuberculosis (MTB) with full sensitivity to first-line anti-TB medications.

There is no consensus on the optimal treatment of chest wall tuberculosis. The general role is for the medical treatment, in the form of anti-tuberculosis medications, with a six-month regimen being sufficient in most cases. Surgical intervention may be needed in cases of extensive tissue damage, the presence of draining sinuses, or failure of medical treatment. $(16,17)$

\section{Conclusion:}

Chest wall tuberculosis is a rare entity that may occur even in an immunocompetent patient. A high index of suspicion is required to make an early diagnosis and overcome the diagnostic dilemma. Using a simple non-invasive procedure in the form of needle aspiration will get the diagnosis in most cases. Early and prompt treatment will prevent the occurrence of complications.

\section{References :}

1- Bloom BR, Atun R, Cohen T, et al. tuberculosis. In: Holmes KK, Bertozzi S, Bloom BR, et al., editors. 
Major Infectious Diseases. 3rd edition. Washington (DC): The International Bank for Reconstruction and Development / The World Bank; 2017 Nov 3. Chapter 11.

2- Organization WH. Tuberculosis surveillance and monitoring in Europe 2019: 2017 data. 2019;

3- Wiebe ER, Elwood RK (1991) Tuberculosis of the ribs-a report of three cases. Respir Med 85: 251-253.

4- Dheda K, Barry CE 3rd, Maartens G. Tuberculosis. Lancet. 2016 Mar 19;387(10024):1211-26. DOI: 10.1016/S0140-6736(15)00151-8. Epub 2015 Sep 13. Erratum in: Lancet. 2016 Mar 19;387(10024):1162. Erratum in: Lancet. 2016 Mar 19;387(10024):1162. Erratum in: Lancet. 2016 May 21;387(10033):2092. PMID: 26377143.

5- Verver S, Bwire R, Borgdorff MW. Screening for pulmonary tuberculosis among immigrants: estimated effect on severity of disease and duration of infectiousness. Int J Tuberc Lung Dis. 2001 May;5(5):419-25. PMID: 11336272 .

6- Global tuberculosis report 2019 (WHO)

7- Lee SH, Abramson SB (1996) Infections of the musculoskeletal system by M.tuberculosis. In: Rom WN, Garay SM, eds. Tuberculosis. New York: Little Brown, Pp. 635-644.

8- H. E. Burke, "The pathogenesis of certain forms of extrapulmonary tuberculosis; spontaneous cold abscesses of the chest wall and Pott's disease," The American Review of Tuberculosis, vol. 62, no. 1 B, pp. $48-67,1950$.

9- Laube S, Clarke J, Parikh DH, Moss C. Tuberculous cold abscess resembling a lymphatic malformation. Clin Exp Dermatol. 2005 Sep;30(5):611-3. DOI: 10.1111/j.1365-2230.2005.01883.x. PMID: 16045721.

10- Narang M, Dwivedi A, Narang S, Mehrotra GSternal Tuberculosis: An Uncommon Presentation.JCR 2011;1:9-11.

11- Anand P MD, Sarin N MD. Isolated Sternal Tuberculosis Presenting as a Chest Wall Abscess: A Case Report. Iran J Med Sci. 2018;43(4):440-443.

12- Grover SB, Jain M, Dumeer S, Sirari N, Bansal M, Badgujar D. Chest wall tuberculosis - A clinical and imaging experience. Indian J Radiol Imaging. 2011;21(1):28-33. DOI:10.4103/0971-3026.76051.

13- Goyal M, Sharma R, Sharma A, Chumber S, Sawhney S, Berry M. Chest wall tuberculosis simulating breast carcinoma: imaging appearance. Australas Radiol. 1998 Feb;42(1):86-7. DOI: 10.1111/j.14401673.1998.tb00574.x. PMID: 9509615.

14-Shah J, Patkar D, Parikh B, Parmar H, Varma R, Patankar T, et al. tuberculosis of the sternum and clavicle: Imaging findings in 15 patients. Skeletal Radiol. 2000;29:447- 53.

15- C. Piersimoni and C. Scarparo, "Extrapulmonary infections associated with nontuberculous mycobacteria in immunocompetent persons," Emerging Infectious Diseases, vol. 15, no. 9, pp. 1351-1358, 2009.

16- Rajput AK, Rajan KE, Gupta RK, Muttagikar MP. TUBERCULOSIS OF STERNUM. Med J Armed Forces India. 2001;57(4):330-332. DOI:10.1016/S0377- 1237(01)80017-4 ).

17- Nahid P, Dorman SE, Alipanah N, et al. Official American Thoracic Society/Centers for Disease Control and Prevention/Infectious Diseases Society of America Clinical Practice Guidelines: Treatment of DrugSusceptible Tuberculosis. Clin Infect Dis. 2016;63(7):e147-e195. DOI:10.1093/cid/ciw376

\section{Hosted file}

Figure.pdf available at https://authorea.com/users/392685/articles/506465-extrapulmonarytuberculosis-masquerading-as-chest-wall-malignancy-it-just-never-ceases-to-surprise-us

\section{Hosted file}


Table 1.pdf available at https://authorea.com/users/392685/articles/506465-extrapulmonarytuberculosis-masquerading-as-chest-wall-malignancy-it-just-never-ceases-to-surprise-us 